\title{
A Revised Sound Energy Theory Based on a New Formula for the Reverberation Radius in Rooms with Non-Diffuse Sound Field
}

\author{
Higini ARAU-PUCHADES(1), Umberto BERARDI ${ }^{(2)}$ \\ (1) ArauAcustica \\ Barcelona, Spain \\ (2) Department of Architectural Science, Ryerson University \\ Toronto, Canada; e-mail: uberardi@ryerson.ca
}

(received November 9, 2014; accepted November 20, 2014)

\begin{abstract}
This paper discusses the concept of the reverberation radius, also known as critical distance, in rooms with non-uniformly distributed sound absorption. The reverberation radius is the distance from a sound source at which the direct sound level equals the reflected sound level. The currently used formulas to calculate the reverberation radius have been derived by the classic theories of Sabine or Eyring. However, these theories are only valid in perfectly diffused sound fields; thus, only when the energy density is constant throughout a room. Nevertheless, the generally used formulas for the reverberation radius have been used in any circumstance. Starting from theories for determining the reverberation time in nondiffuse sound fields, this paper firstly proposes a new formula to calculate the reverberation radius in rooms with non-uniformly distributed sound absorption. Then, a comparison between the classic formulas and the new one is performed in some rectangular rooms with non-uniformly distributed sound absorption. Finally, this paper introduces a new interpretation of the reverberation radius in non-diffuse sound fields. According to this interpretation, the time corresponding to the sound to travel a reverberation radius should be assumed as the lower limit of integration of the diffuse sound energy.
\end{abstract}

Keywords: reverberation radius, critical distance, non-diffuse sound field, sound energy, revised sound decay theory.

\section{Introduction}

The reverberation radius in a room, or critical distance as it is often called in audio engineering, is the distance from a sound source at which the level of direct sound equals the level of reflected sound (Fig. 1). This distance is an important parameter for the sound perception as by knowing the distance of the listener from a source and the reverberation radius of the room, it is then possible to assess if the direct or diffused sound filed prevails. The reverberation radius is hence highly useful in assessing the reverberance of a room. Generally, a room is defined as "live" when a preponderance of reflected sounds produces a reverberant feeling; on the other hand, if the direct sound is dominant, the room is considered "dry" or "dead". Attempts have been made to express liveness as the ratio of the direct and diffuse energy density, often using the reverberation radius concept. In this way, the comparison of the reverberation radius with the room dimensions allows an easy estimation.

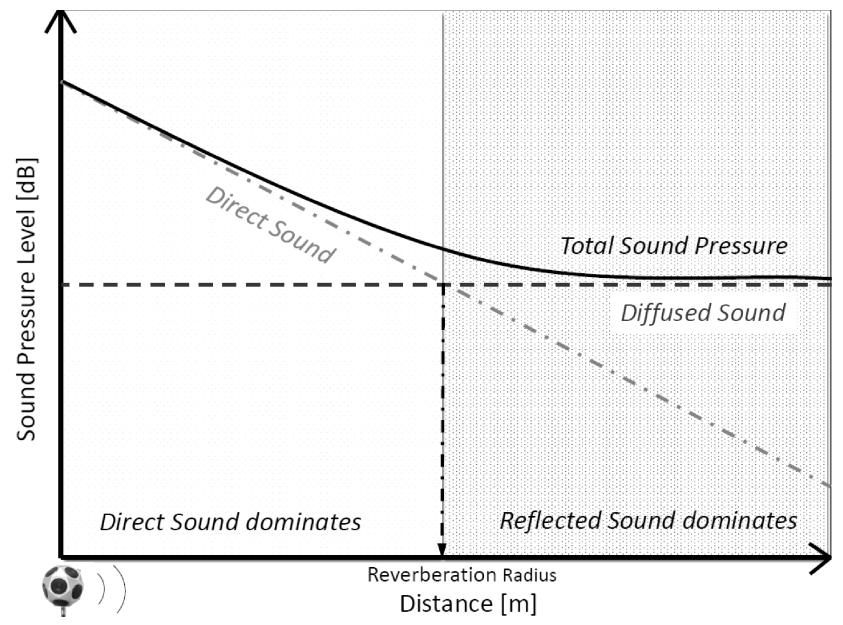

Fig. 1. Sound energy decay and reverberation radius representation. 
In the sound recording field, studies proved that microphones must be located at a distance from the sound source shorter than the reverberation radius during recording (MiJic, MAsovic, 2010). Such a position of the microphone allows recording a signal in which the direct sound prevails, minimizing the effect of the room.

Available formulas to calculate the reverberation radius refers to perfectly sound diffuse fields. According to these formulas, if the sound source is omnidirectional, the value of the reverberation radius $\left(r_{H D}\right)$ is the same in any directions. These formulas are based on the classic theories of SABINE (1922) and EYRING (1930). Both of these theories assume that sound pressure variance is zero, as a consequence a perfectly diffuse sound space with a constant sound energy density. In these cases, the mean free sound path within the room is equal to $4 V / S$, where $V$ is the volume of the room and $S$ is its total surface. BERANEK and NISHIHARA (2014) recently proved that the well-known formula for the mean free path between reflections may be considered valid in most of the rooms, except for halls of unusual shapes such as the Tokyo Opera City hall.

Conversely, in a non-diffuse sound field, sound energy density is not constant. Therefore, the fluctuations in the sound pressure levels depend on the considered direction. A non-uniform distribution of absorption in a room is often the main reason for a nondiffuse sound field. Moreover, other phenomena, normally wave-type, such as resonance, interference, and focalization, may produce privileged sound-wave directions avoiding the sound to diffuse across the volume uniformly.

As a consequence of the non-uniform distribution of the sound energy, the reverberation radius is not constant, and the known formulas for calculating it are not valid anymore. Recent investigations in nonuniform sound fields have revealed that the measured values of the reverberation radius contradicted the expectations formulated according to the classic theories (ŠumaraC-PAVlović, MiJić, 2007; LARSEN et al., 2008; Misić, Masovic, 2010).

The present paper proposes and tests a formula to calculate the reverberation radius in rooms with nonuniformly distributed sound absorption $\left(r_{H N D}\right)$ and test it in some real rooms. The paper is based on a preliminary study presented by ARAU-PUCHADES and BERARDI (2013) at a recent ICA conference, but instead of reporting the measurements taken in a real room by the authors as it was done in that study, in this one, software simulations are included.

This paper is structured in the following way: Secs. 2 and 3 discuss the formulas of the reverberation radius in diffuse and non-diffuse sound fields respectively; Sec. 4 assesses the different formulas in some case studies; Sec. 5 discusses the implications of the different formulas of the reverberation radius on the revised theory of sound decay; finally, Sec. 6 reports concluding remarks.

\section{The reverberation radius in diffuse sound fields}

Sound level intensity is the sum of the direct sound intensity $\left(I_{\text {direct }}\right)$ and diffuse sound intensity $\left(I_{\text {diffuse }}\right)$. Sound pressure level may be determined from the intensity level according to:

$$
\begin{aligned}
L_{p}=L_{I} & =10 \log \left(I_{\text {total }} / I_{\text {ref }}\right) \\
& =10 \log \left(\left(I_{\text {direct }}+I_{\text {diffuse }}\right) / I_{\text {ref }}\right),
\end{aligned}
$$

where $I_{\text {ref }}$ is the reference intensity and is equal to $10^{-12}$ Watts $/ \mathrm{m}^{2}$, whereas the direct intensity can be obtained considering the geometrical spreading of the sound, as:

$$
I_{\text {direct }}=Q W / 4 \pi r^{2},
$$

where $Q$ is the source directivity, $W$ is the sound power of the source, and $r$ is the distance from the source to the receiver. Moreover, the diffuse intensity is equal to:

$$
I_{\text {diffuse }}=4 W / A,
$$

where $A$ is total sound absorption in the room. According to Eyring, the sound absorption is equal to $[-S \ln (1-\alpha)+4 m V]$, where $\alpha$ is the average absorption coefficient among the surfaces, and $m$ is the sound absorption coefficient of the air.

In the classical theory, the diffuse intensity may be also expressed also as:

$$
I_{\text {diffuse }}=25 W(T / V) \text {. }
$$

From Eqs. (1)-(3) the sound pressure level may be expressed as:

$$
L_{p}=L_{w}+10 \log \left(1 / 4 \pi r^{2}+(4 / A)\right),
$$

or

$$
L_{p}=L_{w}+10 \log \left(1 / 4 \pi r^{2}+(25(T / V)) .\right.
$$

When it is reasonable to assume the source as omnidirectional, then the $Q$ term in previous formulas is neglected. Moreover, the absorption in the room reduces to simple multiplication of the surface and the absorption, $A=S \cdot \alpha$, whenever the absorption in the room is particularly low $(\alpha<0.2)$, and the air absorption is insignificant $(m V \approx 0)$. This last requisite is generally valid at frequencies below $4 \mathrm{kHz}$ (BERARDI, 2014).

By considering the definition of the reverberation time, the formulas $(4)_{1}$ and $(4)_{2}$ lead to the classic SABINE's formula (1922):

$$
T=0.16 \mathrm{~V} / \mathrm{A} \text {. }
$$


This formula has sometimes been criticized and consequently, many other theories have been proposed to calculate the reverberation time (MilLingTon, 1932; Pujolle, 1975; Kuttruff, 1991). However, one of the limits of the theories alternative to the classic diffuse sound field is that they often underestimate the reverberation time (Mehta, Mulholland, 1976; Bistafa, Bradley, 2000; Ducourneau, Planeau, 2003). For this reason, the Sabine's formula and the diffused sound theory are still widely used.

Knowing the expressions for the direct and diffuse sound pressure levels, it is possible to obtain the formula for the reverberation radius in a diffuse field $\left(r_{H D}\right)$. This comes from equaling the sound intensity in $(2)$ and the reflected sound intensity in $(3)_{1}$ or $(3)_{2}$ :

$$
\begin{aligned}
& r_{H D}=(A / 16 \pi)^{0.5}, \\
& r_{H D}=((0.01 / \pi)(V / T))^{0.5} .
\end{aligned}
$$

As it is evident from the expressions in (6), the reverberation radius increases when the total absorption increases or when the reverberation time decreases. The Eqs. $(6)_{1}$ and $(6)_{2}$ allow to calculate the distance between a sound source and receivers where the direct field sound level emitted from the source is equal to the level in the reverberated sound field. In a perfectly diffuse sound field and with an omnidirectional source, the reverberation radius does not depend on the direction, and it shapes a sphere at equal distance $\left(r_{H D}\right)$ from the source. This means that at any point within the room at which the sound source is placed, there will be a spherical surface with radius $r_{H D}$. However, a perfectly diffuse sound field is difficult to create, and also rooms with diffuse sound fields, such as sports halls (GERRETSEN, 2006) or large reverberant churches (BERARDI, 2012; 2014), cannot be considered perfectly diffuse. Generally, as a rule of thumb, any space in which some surfaces have an absorption coefficient greater than 0.2 may be difficult to consider perfectly diffuse. This means that in rooms such as recording studios or rooms acoustically designed for speech (classrooms or auditoriums) the sound field is rarely diffuse. The room shape also plays an important role over the diffusiveness of the sound field (BERANEK, Nishinara, 2014). Finally, it is evident that the formulas for calculating the reverberation radius in (6) are hence invalid in most of the real rooms.

\section{Reverberation radius in non-diffuse sound fields}

The first non-diffuse sound field theory was proposed by Fitzroy over 50 years ago (1959). His proposition became established through an intuitive formula followed by repeated experimentations. His theory was based on an earlier idea put forward by Bagenal who stated: "Reverberation in a rectangular room really consists of three sets of inter-reflections set up between the three pairs of opposite surfaces. It is important that these three reverberation times should be roughly of equal length as one smooth tone" (BAGENAL, 1941). However, Bagenal did not indicate which type of mean should be taken into account for the calculation of the reverberation time. Fitzroy's proposal for the reverberation time in rectangular rooms with non-diffuse sound field considered the arithmetical average, weighted by the fraction of area, of the three reverberation times calculated in each main direction: those connecting the floor and the ceiling, the right and left-hand lateral surfaces, and the front-back surfaces.

In 1988, ARAu-PuCHADES attracted by the intuition of the Fitzroy's formula, sought to explore it further. He introduced a model to calculate the reverberation time for the case of asymmetrical distribution of absorption, assuming that the reverberation decay is a hyperbolic process (ARAu-Puchades, 1988). This decay was supposed to be a superposition of three contributions: initial decay, first and second linear portion of the decay. This led him to demonstrate that the reverberation time for a non-diffuse field may be expressed as the geometric average of three reverberation times in each direction, weighted by the fraction of the area. Arau worked with a logarithmic-normal distribution of the decay coefficients which were proportional to the absorption coefficients in the three directions $\left(\alpha_{x}, \alpha_{y}, \alpha_{z}\right)$. Through this approach, he assumed a distribution of the absorption that complies with the Gauss's normal law of the mean free path. He then proposed to use the average absorption of each pair of opposite walls as the absorption coefficients used in his formula (ARAu-Puchades, 1988). The resulting reverberation time is hence:

$$
T=T_{x}^{S x / S} \cdot T_{y}^{S y / S} \cdot T_{z}^{S z / S}
$$

where each factor $T_{i}$ (for $i=x, y, z$ ) is:

$$
T_{i}=0.16 \mathrm{~V} / A_{i}=0.16 \mathrm{~V} /\left(-S \ln \left(1-\alpha_{i}\right)+4 m V\right)
$$

and the absorptions $S_{i}$ in each direction are:

$$
\begin{aligned}
A_{x}= & {\left[-S \ln \left(1-\alpha_{x}\right)+4 m V\right], } \\
& \text { where } \alpha_{x}=\left(S_{x 1} \alpha_{x 1}+S_{x 2} \alpha_{x 2}\right) / S_{x}, \\
A_{y}= & {\left[-S \ln \left(1-\alpha_{y}\right)+4 m V\right], } \\
& \text { where } \alpha_{y}=\left(S_{y 1} \alpha_{y 1}+S_{y 2} \alpha_{y 2}\right) / S_{y}, \\
A_{z}= & {\left[-S \ln \left(1-\alpha_{z}\right)+4 m V\right], } \\
& \text { where } \left.\alpha_{z}=S_{z 1} \alpha_{z 1}+S_{z 2} \alpha_{z 2}\right) / S_{z} .
\end{aligned}
$$

Finally, the expression of the reverberation time in (7) may be re-written as:

$$
\begin{aligned}
T= & \left(0.16 V / A_{x}\right)^{S x / S} \cdot\left(0.16 V / A_{y}\right)^{S y / S} \\
& \cdot\left(0.16 V / A_{z}\right)^{S z / S} .
\end{aligned}
$$


The Arau's formula shows that Fitzroy's theory was not completely correct, as it was only valid when the reverberation times in each direction $\left(T_{x}, T_{y}, T_{z}\right)$ are equal, or approximately equal. A tendency for coincidence of the Fitzroy's formula with the Sabine's and Eyring's formulas may occur depending on the closeness of the average absorption coefficients in every direction. However, whenever the reverberation times are well differentiated among the directions, then the Fitzroy's formula diverges significantly from theoretical results as recently shown in some round robin tests (Mehta, Mulholland, 1976; Istafa, Bradley, 2000; Ducourneau, Planeau, 2003).

The formula (10) covers diffuse and non-diffuse sound fields, and appears as a general formulation of the theory of reverberation. In fact, in case of a uniform absorption distribution within the space, it coincides with Sabine's or Eyring's formulas. From Eq. (10), it is possible to calculate the reverberation radius in each direction similarly to the expression (6):

$$
r_{H N D i}=\left(Q A_{i} / 16 \pi\right)^{0.5} .
$$

In (11), the reverberation radius in each direction increases as the equivalent absorption in that direction increases. From this equation, it is possible to calculate the reverberation time as a product of terms of reverberation for the facing surfaces, each one expressed as a function of the reverberation radius in that direction:

$$
\begin{aligned}
T= & \left(0.01 V / \pi r_{H N D x}^{2}\right)^{S x / S} \cdot\left(0.01 V / \pi r_{H N D y}^{2}\right)^{S y / S} \\
& \cdot\left(0.01 V / \pi r_{H N D z}^{2}\right)^{S z / S} \\
T= & \left(0.01 V / \pi r_{H N D}^{2}\right)
\end{aligned}
$$

where $r_{H N D}=r_{H N D x}^{S x / S} \cdot r_{H N D y}^{S y / S} \cdot r_{H N D z}^{S z / S}$.

The reverberation radius for a non-diffuse sound field $r_{H N D}$ in a room is finally obtained as:

$$
\begin{aligned}
r_{H N D}= & (Q A / 16 \pi)^{0.5}, \\
& \text { where } A=A_{x}^{S x / S} \cdot A_{y}^{S y / S} \cdot A_{y}^{S z / S}, \\
r_{H N D}= & ((Q 0.01 / \pi)(V / T))^{1 / 2} .
\end{aligned}
$$

It is evident that if $A_{x}, A_{y}$, and $A_{z}$ tend to be equal, which corresponds to have similar absorption in the three directions, then $r_{H N D} \approx r_{H D}$. In this case, the reverberation radius in (11) equals the formulas for diffuse sound fields in (6).

\section{Case study analysis of the reverberation radius in non-diffuse sound fields}

The results of a comparison among values of the reverberation radius according to different formulas are reported in this section.
Figure 2 shows the configurations of the rooms used in the experimental inter-comparison among theories for non-diffuse spaces carried out by MEHTA and MuLHOLLAND (1976). This study was the first comparative test about asymmetric distribution absorption in a room. The room had the following dimensions: $4.5 \mathrm{~m}$ length, $2.7 \mathrm{~m}$ width, and $2.4 \mathrm{~m}$ height. Its volume was $29.16 \mathrm{~m}^{3}$. Five of the Mehta-Mulholland's cases have been investigated. Moreover, the simple space, with no absorptive panels, was considered. This case was used to verify the accuracy of the models, and it is here referred as case 0 . In the other five configurations, an absorptive material was used to add non-uniform absorption to the room. The surface not covered of this material was simulated as normal gypsum plaster. The investigated rooms are represented in Fig. 2: case 1, absorption on the long walls; case 2, absorption on one long wall; case 3, absorption on the floor and the two short walls; case 4, absorption on the floor and on one short wall; case 5 , absorption on three mutually perpendicular surfaces.
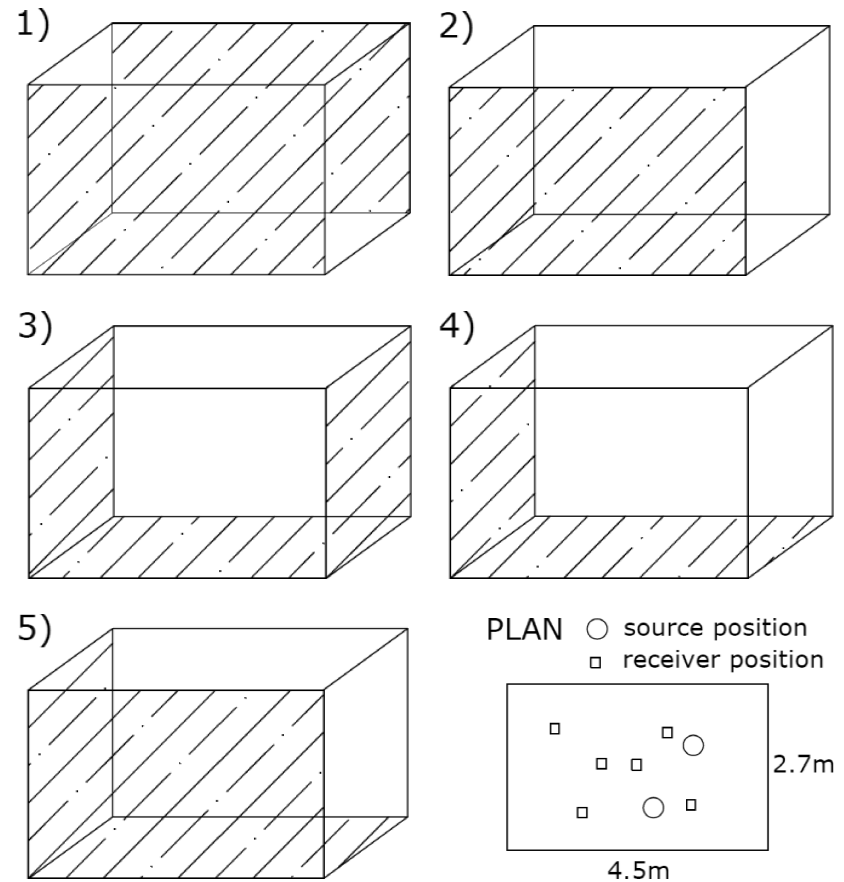

Fig. 2. Configurations of room simulated in this paper: marked walls indicate absorptive surfaces.

\subsection{Acoustic simulation setup}

The software CATT-Acoustics v.8 was used for the simulation (DALENBÄCK, 2008). This software is a room acoustics prediction program based on a mixed algorithm. Firstly, it performs a ray tracing algorithm analysis according to the image source method and then, a diffuse reflection algorithm allows studying the late decay of the sound (DALENB̈̈CK, 2008). The software utilizes a randomized tail-corrected cone-tracing 
Table 1. Material properties selected for the CATT simulations.

\begin{tabular}{|c|c|c|c|c|c|c|c|c|c|c|c|c|}
\hline & \multicolumn{6}{|c|}{ Absorption coefficient } & \multicolumn{6}{|c|}{ Scattering coefficient } \\
\hline & $125 \mathrm{~Hz}$ & $250 \mathrm{~Hz}$ & $500 \mathrm{~Hz}$ & $1 \mathrm{kHz}$ & $2 \mathrm{kHz}$ & $4 \mathrm{kHz}$ & $125 \mathrm{~Hz}$ & $250 \mathrm{~Hz}$ & $500 \mathrm{~Hz}$ & $1 \mathrm{kHz}$ & $2 \mathrm{kHz}$ & $4 \mathrm{kHz}$ \\
\hline Absorptive surfaces & .50 & 0.60 & 0.70 & 0.80 & 0.80 & 0.80 & .35 & 0.40 & 0.50 & 0.50 & 0.60 & 0.65 \\
\hline No absorptive surfaces & .03 & 0.03 & 0.05 & 0.05 & 0.07 & 0.07 & .35 & 0.40 & 0.50 & 0.50 & 0.60 & 0.65 \\
\hline
\end{tabular}

that combines features of both specular cone-tracing and standard ray tracing (Summers et al., 2005).

For diffuse-reflection order equal to one, a number of elementary sources are created on each diffusing surface. Power is radiated from the surface according to the Lambert's cosine law, and is proportional to $s(1-\alpha)$, where $s$ is the scattering coefficient of the surface and $\alpha$ is the absorption coefficient. Similarly, the powers of first-order and second-order specular reflections are reduced by $(1-s)(1-\alpha)$.

For the direct sound and first-order specular reflections, values at frequencies between the input octaveband centre frequencies are found by interpolation, using a cubic-spline method. For first-order diffuse reflection, the software creates many secondary sources whose number is automatically decided proportionally to the scattering of the material. All the reflections both of the first-order and higher-order reflections are divided by octave-band filters before being summed together. The room-prediction algorithm includes phase in order to predict impulse response. The reflectionpath phase calculation method depends on the reflection order. Main advantages of CATT-Acoustics are that room surfaces can be modelled with frequencydependent absorption and scattering coefficients, and that cone-tracing is performed independently for each frequency band. More details about the software are available in Hodgson et al. (2008) and BERARDI (2014).

Geometrical characteristics of the room were simulated similarly to the study in MEHTA and MulHOLLAND (1976) to help comparison of the results. In this way it was possible to compare the simulated results with measured ones in that study. Table 1 reports the material properties adopted in the simulations.

\subsection{Results}

The results of the reverberation time with the different formulas, Sabine's, Eyring's and Arau's ones, are shown in Fig. 3 for the frequency band of $1 \mathrm{kHz}$. This frequency band was considered representative after having verified that a similar pattern occured for other frequency bands too. Figure 3 also shows the reverberation radii for the six cases according to the respective formulas for each theory. In general, the simulated values for the reverberation radius were lower than the predicted through the formulas at lower frequency (particularly in the low frequency band of $125 \mathrm{~Hz}$ ) and a)

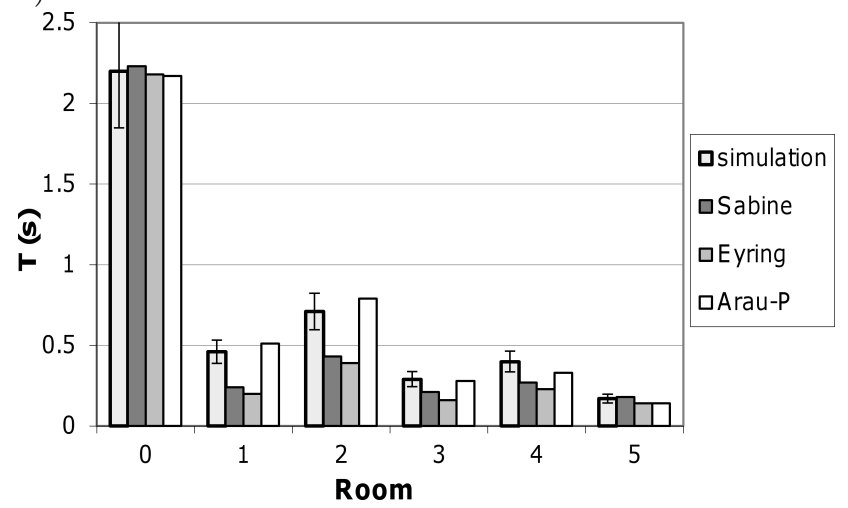

b)

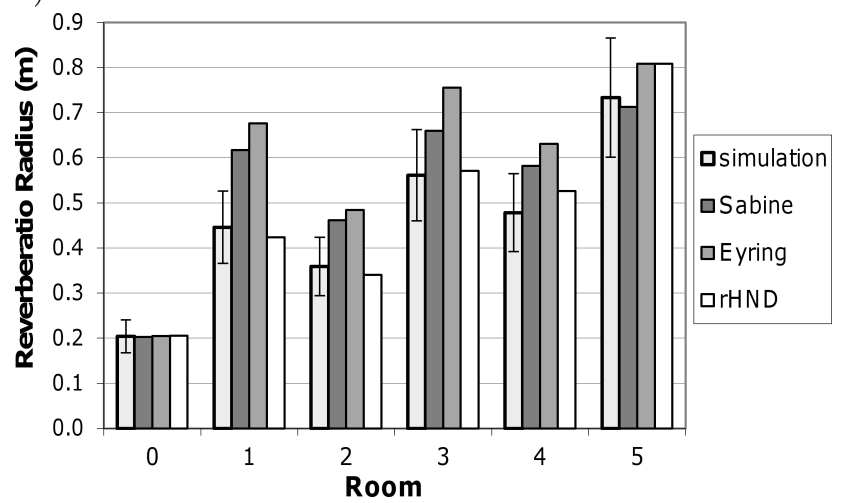

Fig. 3. Reverberation time and reverberation radius simulated or predicted with the Sabine's or Eyrings' formulas, or with the $\mathrm{r}_{H N D}$ formula proposed in this paper.

were higher at higher frequencies. The results have not been considered reasonable at such low frequency band given the intrinsic limitations of ray-tracing software simulations at low frequency (BERARDI, 2014), given the strong effect of room modes (SAKUMA, 2012); consequently, the results at low frequencies are not considered in this paper.

The standard deviations report the average among six different receiver positions in the simulations and two source positions in each room.

Results of the reverberation radius show that the error committed in the estimation with the Sabine's or Eyring's formula is considerable, being on average $18.5 \%$ and $28.9 \%$ of the simulated value respectively. The estimation with the new formula for $r_{H N D}$ is much more accurate, and it results in an average error of $2.5 \%$ only. In particular, it is important to underline that the reverberation radius calculated with the Sabine's or Eyring's formulas often exceeds the sim- 
ulated values. Reasons for this were firstly given by BARRON and LEE in their revised theory (1988), where they showed that the level of the reflected sound decreases with the distance from the sound source.

A description of the results in the different cases is as follows:

- in case 0 , the different formulas for the reverberation time and the reverberation radius predict similar results; hence, all the formulas may be considered accurate with high diffusiveness;

- in case 1, the Arau-P's formula is significantly more accurate than the classic formulas. The formula proposed in $(14)_{1}$ resulted in an error of a few cm, whereas the errors with the Sabine's and Eyring's formula were $0.47 \mathrm{~m}$ and $0.61 \mathrm{~m}$ respectively. This demonstrates the limitation of the classic theories, in rooms with a strongly nonuniform distribution of absorption;

- in case 2, with absorption on just one long wall, the simulated reverberation time is $0.19 \mathrm{~s}$ longer than in case 1 , and the simulated reverberation radius decreased from $0.42 \mathrm{~m}$ to $0.36 \mathrm{~m}$. These differences were partially predicted by the classic formulas, but only the result of the new $r_{H N D}$ is accurate (the error in the reverberation radius estimate was $0.02 \mathrm{~m}$ with the formula in $(14)_{2}$ and $0.20 \mathrm{~m}$ and $0.12 \mathrm{~m}$ with the Sabine's and Eyring's formula respectively);

- in case 3 , the reverberation time reduces to $0.29 \mathrm{~s}$ which is accurately predicted by Arau and underpredicted by the other formulas. However, the percentage differences between the methods are larger for the values of the reverberation radius than for the reverberation time;

- in case 4 , the classical models underestimate the reverberation time value and overestimate the reverberation radius, whereas the Arau's formula for the reverberation time results similar to the simulated one and the reverberation radius in $(14)_{1}$ gives an error of only $1 \mathrm{~cm}$;

- in case 5, the Arau's and Eyring's formulas produce less accurate prediction of the reverberation time than the Sabine's formula; this reflects on the estimation of the reverberation radius where a $2 \%$ error in the estimation with the Sabine's formula occurs, while the other formulas give an error of $10 \%$.

Previous results suggest some useful considerations. Firstly, when the reverberation time is particularly long, the different formulas generally approach more similar values (Fig. 4); as a consequence the reverberation radii calculated with different formulas were particularly close. On the other side, the classic formulas should not be used in case of strongly nonhomogenous sound absorption distribution. This is for example the case of classrooms and conference halls with absorbing surfaces on the ceiling of on one lateral wall. Similarly, the classic formulas do now seem to be adequate when most of the absorption is concentrated in one direction, which again is sometimes the case for architectural symmetrical reasons.

Finally, Fig. 4 visualizes clearly the value of the new formula for calculating the reverberation radius over the classical ones.

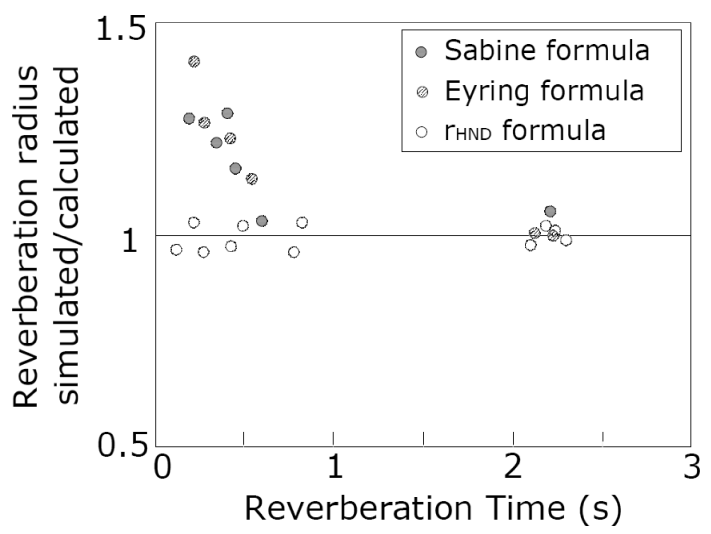

Fig. 4. Simulated over calculated reverberation radius using different formulas (classic Sabine's or Eyrings' formulas and the $r_{H N D}$ formula proposed in this paper) for different reverberation times of the room.

\section{Interpreting the reverberation radius within the "revised" theory}

According to the classic theory the sound strength should not vary considerably at sufficient distance from the source where the energy of the reflected sound field, equally distributed throughout the space, is dominant. However, BARRON and LEE (1988) showed that the reflected sound energy may significantly fall off with increasing source-receiver-distance. Barron and Lee found out that the sound level decay was linear soon after the direct sound in the majority of the halls and the reflected sound level decreased with increasing source-receiver distance. This is due to the fact that receivers closer to the source not only receive a higher level of direct sound, but also higher levels of early reflections because these have travelled shorter distances. Thus, they proposed a model based on the following assumptions: the direct sound is followed by linear level decay at a rate corresponding to the reverberation time; the instantaneous level of the late decaying sound is uniform throughout the space; the time $t_{0}$ corresponds to the time the signal is emitted from the source, therefore the direct sound reaches a point at a distance $r$ from the source after a time $t_{D}=r / c$. In this way the integrated energy decreases when the source-receiver distance increases, while the early/late reflected energy ratio remains constant; the integrated value for the reflected sound level is assumed to be, 
at $r=0$, equal to the value predicted by the classic theory (BERARDi et al., 2009). According to the Barron and Lee's revised theory of sound decay, the sound energy may be calculated as

$$
G=10 \log _{10}\left(100 / r^{2}+31200 \cdot T / V \cdot e^{(-0.04 \cdot r / T)}\right)
$$

The exponential term $(-0.04 \cdot r / T)$ marks the difference between the classic and the revised theory. It accounts for the fact that the linearly decaying reflected sound, which is assumed to have a uniform instantaneous level at late time, cannot start before the arrival time of the direct sound $t_{0}=r / c$, thus yielding a refined integration limit for the calculation of the total reflected sound level.

Although applying the revised theory to concert halls markedly improves the prediction accuracy compared to classic theory, the revised theory still has some limitations (VORLÄNDER, 1995; BERARDI et al., 2009). In particular, there has been significant discussion in the last years about the appropriate starting time $t_{0}$ for the integration of the reverberant field. Vorländer suggested that the integration should not start at the arrival time of the direct sound but at the arrival time of the first order reflections, and he showed that the lower limit of integration is identical to the mean free path (VORLÄNDER, 1995). Another reasonable assumption for the starting time of the integration has been the direct sound delay plus the delay of the first order reflections (ITDG). Barron acknowledges that considering the ITDG might be beneficial and possibly offer more accurate predictions, but he points out that this would require additional input parameters such as consideration of the shape and geometry of the hall and the exact source and receiver position (VORLÄNDER, 1995). In addition, he makes clear that irrespective of the choice of $t_{0}$, precise agreement from this theory cannot be expected since using continuous integration of the energy fractions of the reflected sound cannot account for the discrete character of the early reflections. In fact, several other methods with no continuous integration have been developed in recent years. The reader can refer to BERARDI et al. (2009) for a review of no continuous integration methods.

Interpreting what happens to the sound field after the emission of a sound, it is clear that initially only the direct sound exits. Then, the reflection of the sound on the surfaces of the room generates distinctive reflections. However, the direct sound level is higher and prevents perceiving the reverberated sound field for a while. This means that, during a lapse of time that can be approximated to the time required by the sound to travel a reverberation radius, our hearing cannot hear the reverberated field although the first sound reflections have already been occurred on the walls of the room. Consequently, in a highly reverberant room (long $T$ corresponds to short $r_{H}$ ) the reverberated field is perceived almost instantly; reversely, if the space is dry (short $T$ corresponds to long $r_{H}$ ), it takes longer to hear the reverberated sound field.

As we stated earlier, a useful way to interpret this is through considering that at shorter distances than the reverberation radius, the direct sound dominates, and at longer distances than the reverberation radius, the diffuse sound field dominates (ARAU-PUCHADES, 2012). This means that in a point within a reverberation radius distance, the listener will perceive clearly the direct sound, that then will start decreasing, and after a while the diffuse sound field will become perceptible. In this way, a longer time separates the direct and the diffused sound field (it is useful to consider the analogy between being at a distance shorter than the reverberation radius and having to wait longer to have an equal sound level between direct and diffuse sound level). Reversely, when the listener is at a distance from the source longer than the reverberation radius, the time to wait for the balancing between direct and diffuse sound levels is shorter.

According to this new interpretation of the reverberation radius, this may be considered for assessing the lower limit of integration of the diffuse sound energy. The diffused sound energy can hence be written as:

$$
\stackrel{\infty}{I}=(w / V) \int_{t_{d}}^{\infty} e^{-\left(c\left(t-t_{H}\right) / 4 V\right) A} \mathrm{~d} t,
$$

where the lower limit is $t_{d}=t-t_{H}$, for $t_{d} \geq 0$, with $t_{H}$ the time corresponding to the sound to travel a reverberation radius. The expression in (1) allows rewriting the formulas for the diffuse intensity in (3) as:

$$
\begin{aligned}
& I_{\text {diffuse }}=(4 W / A) \cdot e^{-\left(\left(r-r_{H}\right) / 4 V\right) A}, \\
& I_{\text {diffuse }}=25 W(T / V) \cdot e^{-0.04\left(r-r_{H}\right) / T} .
\end{aligned}
$$

\section{Conclusions}

Before this paper, the concept of reverberation radius was only considered and assessed according to the classic theory of diffuse sound fields. Reversely, in this paper a new formula to estimate the reverberation radius in rooms with no-uniform absorption has been proposed. Case studies have proved the validity of the formula of the reverberation radius for non-uniformly distributed sound absorption. Finally, this paper has shown that the reverberation radius may be used to correct the diffuse intensity in the revised theory, where the lower limit of integration of the diffuse sound energy may be assumed equal to the time required to the sound to travel a reverberation radius.

The investigation of the reverberation radius in real rooms with non-diffuse sound field and non-box shape remains an important field to research. One of the interesting aspects related to the reverberation radius is related to its measurement technique and the relative 
uncertainty. A seminal work on this topic was published by JETZT (1979). He compared the standard deviation method and the asymptotic total energy decay method, and showed that the standard deviation method is more useful because it uses information from measurements made close to the sound source, while the total energy method needs information from distant measurements, and because it determines the reverberation radius in each point independently, while the total energy method is based on the often unrealistic assumption that the reverberant energy is uniform everywhere. However, recently MiJić and Masovic (2010) have confirmed how difficult is an accurate estimation of the reverberation radius and have encouraged further studies in this field. Future study should hence focus on assessing in real cases the limits of validity of the formula proposed in this paper and the tradeoff between the room shape and the assumption of existence of a diffuse sound field.

\section{References}

1. Arau-Puchades H. (1988), An improved reverberation formula, Acustica, 65, 163-180.

2. Arau-Puchades H. (2012), Sound Pressure Level in Rooms: Study of steady state intensity, total sound level, reverberation distance, a new discussion of steady state intensity and other experimental formulae, Building Acoustics, 19, 3, 205-220.

3. Arau-Puchades H., Berardi U. (2013), The reverberation radius in an enclosure with asymmetrical absorption distribution, Proceedings of Meetings on Acoustics, 19, 015141.

4. Bagenal H. (1941), Practical Acoustics and Planning Against Noise, Ed. Methuen.

5. BARRon M., LeE L.J. (1988), Energy relations in concert auditoriums, J. Acoust. Soc. Am., 84, 618-628.

6. Beranek L.L., Nishinara N. (2014), Mean-free-paths in concert and chamber music halls and the correct method for calibrating dodecahedral sound sources, J. Acoust. Soc. Am., 135, 1, 223-230.

7. Berardi U. (2012), A double synthetic index to evaluate the acoustics of churches, Archives of Acoustics, 37, 4, 521-528.

8. Berardi U. (2014), Simulation of acoustical parameters in rectangular churches, J. Build. Perf. Simul., 7, $1,1-16$.

9. Berardi U., Cirillo E., Martellotta F. (2009), A comparative analysis of energy models in churches, J. Acoust. Soc. Am., 126, 4, 1838-1849.

10. Bistafa S.R., Bradley J.S. (2000), Predicting reverberation times in a simulated classroom, J. Acoust. Soc. Am., 108, 1721-1731.

11. Dalendä̈CK B.I. (2008), CATT-Acoustic v8 user's manual, CATT, Mariagatan 16A, 41471 Gothenburg, Sweden.
12. Ducourneau J., Planeau V. (2003), The average absorption coefficient for enclosed spaces with non uniformly distributed absorption, Applied Acoustics, 64, 845-862.

13. EyRING C.F. (1930), Reverberation time in dead rooms, J. Acoust. Soc. Am., 1, 217-241.

14. Fitzroy D. (1959), Reverberation formula which seems to be more accurate with non-uniform distribution of absorption, J. Acoust. Soc. Am., 31, 893-897.

15. Gerretsen E. (2006), Estimation Methods for Sound Levels and Reverberation Time in a Room with Irregular Shape or Absorption Distribution, Acta Acustica united with Acustica, 92, 797-806.

16. Hodgson M., York N., Yang W., Bliss M. (2008), Comparison of Predicted, Measured and Auralized Sound Fields with Respect to Speech Intelligibility in Classrooms Using CATT-Acoustic and ODEON, Acta Acustica united with Acustica, 94, 6, 883-890.

17. Jetzt J.J. (1979), Critical distance measurement of rooms from the sound energy spectral response, J. Acoust. Soc. Am.,65, 5, 1204-1211.

18. Kuttruff H. (1991), Room Acoustics, Elsevier Applied Science, London.

19. Larsen E., Iyer N., Lansing C.R., Feng A.S. (2008), On the minimum audible difference in directto-reverberant energy ratio, J. Acoust. Soc. Am., 124, 450-461.

20. Mehta M.C., Mulholland K.A. (1976), Effect of non-uniform distribution of absorption on reverberation, Journal of Sound Vibration, 46, 2, 209-224.

21. Misıć M., Masovic D. (2010), Reverberation Radius in Real Rooms, Telfor Journal, 2, 2, 86-91.

22. Millington G. (1932), A modified formula for reverberation, J. Acoust. Soc. Am., 4, 69-82.

23. Pujolle J. (1975), Nouvelle formule pour la dure'de reverberation, Revue d'Acoustique, 19, 107-113.

24. Sabine W.C. (1922), Collected Papers, Dover Pub, New York.

25. Sakuma T. (2012), Approximate theory of reverberation in rectangular rooms with specular and diffuse reflections, J. Acoust. Soc. Am., 132, 4, 2325-2336.

26. Šumarac-Pavlović D., Mijić M. (2007), An insight into the influence of geometrical features of rooms on their acoustic response based on free path length distribution, Acta Acustica, 92, 6, 1012-1026.

27. Summers J.E., Torres R.R., Shimizu Y., DALENBÄCK B.I. (2005), Adapting a randomized beam-axis-tracing algorithm to modeling of coupled rooms via late-part ray tracing, J. Acoust. Soc. Am., 118, 3, 1491-1502.

28. VORLÄNDER M. (1995), Revised relation between the sound power and the average sound pressure level in rooms and consequences for acoustic measurements, Acustica, 81, 332-343. 